\title{
Motor cortical mapping of proximal upper extremity muscles following spinal cord injury
}

\author{
Brenda Brouwer and Diana H Hopkins-Rosseel \\ School of Rehabilitation Therapy, Faculty of Medicine, Queen's University, Kingston, Ontario, Canada, K7L 3 N6
}

\begin{abstract}
Focal transcranial magnetic stimulation was used to map the motor cortical representations of the relaxed and gently contracted biceps brachii, deltoid and triceps muscles in 22 subjects comprised of 12 controls, five subjects with complete and five with incomplete cervical spinal cord lesions (SCI). Motor evoked potentials (MEPs) were rarely observed during the resting condition (3/30 muscles tested; SCI group) which precluded detailed analysis of these data. With background facilitation, the mean number of scalp stimulation sites producing MEPs varied according to muscle $(P<0.001)$; biceps yielded the largest maps and triceps the smallest. The cortical representations of proximal upper extremity muscles were largest for the control group and smallest for the incomplete SCI group although differences were not significant $(P>0.09)$. The optimal site of stimulation (that which produced the largest MEP) was always surrounded by an area producing submaximal MEPs, but was variable across subjects and groups. There was extensive overlap in the motor cortical representation areas corresponding to the three muscles of interest. Following maximal intensity stimulation at the optimal site, the mean MEP amplitudes (normalized) were largest for the biceps muscle and smallest or absent in triceps $(P<0.02)$. No differences were detected between groups $(P>0.50)$. The threshold stimulus intensity was highest for those with incomplete SCI and lowest amongst control subjects $(P<0.05)$, with biceps then deltoid muscles generally having lower thresholds than triceps $(P<0.001)$. The findings suggest that cortical map areas and MEP characteristics are not significantly altered in gently contracting muscles innervated by nerve roots rostral to the lesion. Only activation thresholds are higher following SCI, particularly incomplete lesions, although there is no apparent association with sensorimotor function. The inability to elicit MEPs in the relaxed muscles of patients with SCI fail to support previous reports of expanded motor cortical representations associated with muscles innervated by roots rostral to the lesion.
\end{abstract}

Keywords: spinal cord injury; corticospinal tract; motor cortical mapping; magnetic stimulation

\section{Introduction}

Focal transcranial magnetic stimulation provides a reliable means of non-invasively mapping the motor cortical representations of limb muscles. ${ }^{1}$ The technique is sufficiently sensitive to reveal mediolateral somatotopic progression on the scalp of proximal to distal muscles in a manner consistent with the known representation of the limb on the motor cortex. ${ }^{2,3}$ The areas of the representations of muscles are consistent with findings obtained following direct cortical stimulation, ${ }^{4,5}$ yet evidence suggests that reorganization may occur in response to altered afferent input or neurological trauma.

The cortical representation of the first dorsal interosseous muscle of the reading finger in long-term

Correspondence: B Brouwer
Braille readers is abnormally large; apparently taking over areas normally associated with other fingers. ${ }^{6}$ In individuals with an acquired limb amputation, muscles proximal to the stump occupy an enlarged motor cortical area and are associated with higher than normal amplitude motor evoked potentials (MEPs). ${ }^{7-9}$ Central lesions have also been associated with dramatic changes in the motor map although the evidence is extremely limited. Following complete spinal cord transection the corticospinal projections to muscles whose function has been spared are reportedly more extensive than those to homologous muscles in control subjects. ${ }^{10,11}$ Levy et al ${ }^{10}$ inferred from their results from two subjects with quadriplegia that the motor cortical projection system reorganizes such that cortical areas previously associated with muscles innervated by roots caudal to the lesion are taken over by the most rostrally spared muscle. It is 
suggested that the loss of sensory input likely contributes to this reorganization. By extension, one might expect that changes may not be as robust when there exists varying degrees of sensory and motor sparing below the lesion level. The extent of any changes in motor cortical output following incomplete spinal cord lesion (SCI) is not known.

Following stroke, the patterns and degree of cortical reorganization is quite variable depending on the extent of the lesion, its location, and the degree of motor recovery. ${ }^{12,13}$ Using positron emission tomography Weiller et $a l^{12}$ showed that the recruitment of additional sensorimotor areas was associated with motor recovery. Clinically then, changes to motor maps may be the substrate for functional recovery in paretic limbs although this has not been demonstrated using transcranial magnetic stimulation.

This study characterizes the motor cortical maps associated with proximal upper extremity muscles in adults with complete or incomplete low cervical SCI. These findings and the nature of the motor responses are contrasted with a group of control subjects.

\section{Methods}

Subjects

Studies were carried out on subjects with cervical cord lesions of greater than 2 years duration and on a similarly aged control group. All subjects gave their informed consent following an initial screening to ensure that they had no history of epilepsy, or a cardiac pacemaker, or intracranial metal implants. A clinical neurological examination using the ASIA Impairment Scale $^{14}$ was performed on all subjects with SCI to determine the lesion level and the degree of sensory and motor impairment. The experimental protocol was approved by the local ethics review board.

\section{Stimulation}

A Cadwell MES-10 electromagnetic stimulator was used with a figure-eight coil attachment consisting of two adjacent coils each measuring approximately $4 \mathrm{~cm}$ in diameter. This configuration permits focal stimulation as peak currents are induced under a well defined center. The intensity of stimulation was initially set to $100 \%$ of the stimulator output which corresponds to a peak flux of 2.2 Tesla (manufacturer's specifications).

\section{Experimental procedures}

Subjects were seated comfortably in a chair and wore a modified bathing cap which was pulled taut over the scalp. A grid was centred at the vertex of the head, $\mathrm{Cz}$ (International $10-20$ System $^{15}$ ), with lines intersecting at $1.5 \mathrm{~cm}$ intervals in coronal and sagittal planes. Bipolar surface disc electrodes ( $7 \mathrm{mM}$ in diameter) were placed over the muscle bellies of the biceps brachii, middle deltoid and triceps muscles bilaterally. Electromyographic (EMG) signals were bandpass filtered at $50 \mathrm{~Hz}$ to $2 \mathrm{kHz}$, amplified to $0.5 \mathrm{mV} /$ div and recorded for a $70 \mathrm{~ms}$ period including a $20 \mathrm{~ms}$ pre-stimulus portion. Signals were digitized $(5 \mathrm{kHz}$ per channel) and data stored on a laboratory computer for later analysis.

The figure-eight coil was centred over each grid point over the left hemisphere in turn. At each stimulus site three single maximal intensity stimuli $(100 \%)$ were delivered at $30 \mathrm{~s}$ intervals and the corresponding MEPs were recorded; this constituted one trial. Three trials were performed at each grid point under two conditions: the first with the subject at rest; the second with the subject contracting each of the target muscles in turn at $5 \%$ to $10 \%$ of its maximal voluntary contraction (MVC) determined using a dynamometer. (Subjects who could not activate their triceps muscle against resistance attempted to do so to the best of their ability). The three EMG traces obtained from each trial were

Table 1 Patient demographics

\begin{tabular}{|c|c|c|c|c|c|c|c|c|c|}
\hline Subject & $\begin{array}{c}\text { Age } \\
\text { (years) }\end{array}$ & Sex & $\begin{array}{c}\text { Time since } \\
\text { injury (years) }\end{array}$ & Level & $\begin{array}{l}A S I A^{\mathrm{a}} \\
\text { scale }\end{array}$ & $\begin{array}{l}\text { Elbow } \\
\text { flexors }\end{array}$ & $\begin{array}{c}\text { Wrist } \\
\text { extensors }\end{array}$ & $\begin{array}{c}\text { Elbow } \\
\text { extensors }\end{array}$ & $\begin{array}{l}\text { Finger } \\
\text { flexors }\end{array}$ \\
\hline 1 & 31 & M & 8 & C7 & A & 5 & 4 & 3 & 0 \\
\hline 2 & 32 & M & 10 & C6 (I) & B & 5 & 3 & 1 & 0 \\
\hline 3 & 25 & M & 7 & $\mathrm{C} 7$ & A & 5 & 4 & 3 & 0 \\
\hline 4 & 40 & M & 12 & C6 (I) & B & 4 & 3 & 0 & 0 \\
\hline 5 & 30 & M & 12 & C7 (I) & $\mathrm{C}$ & 5 & 5 & 3 & 2 \\
\hline 6 & 35 & M & 10 & C8 (I) & D & 5 & 5 & 5 & 3 \\
\hline 7 & 41 & $\mathrm{~F}$ & 23 & C6 & A & 4 & 3 & 0 & 0 \\
\hline 8 & 35 & M & 2 & C7 (I) & B & 5 & 4 & 3 & 0 \\
\hline 9 & 42 & M & 18 & $\mathrm{C} 5$ & A & 5 & 2 & 0 & 0 \\
\hline 10 & 36 & M & 14 & C6 & A & 5 & 4 & 0 & 0 \\
\hline
\end{tabular}

${ }^{a}$ ASIA Impairment Scale ${ }^{14} ; \mathrm{A}=$ no motor or sensory function preserved in S4-S5 segments; $\mathrm{B}=$ some sensory preservation below the lesion level through to $\mathrm{S} 4-\mathrm{S} 5 ; \mathrm{C}=$ most key muscles below the lesion level have a grade $<3 ; \mathrm{D}=$ most key muscles below the lesion level have a grade $>3 ; \mathrm{E}=$ normal sensory and motor function. Muscles graded in accordance with the 6 point Medical Research Council system $(0=$ total paralysis; $5=$ normal movement against full resistance $)$ 
superimposed and averaged using commercial software (DataWave Technologies, Colorado, USA). The response amplitude (peak-to-peak), duration, and the onset latency were measured from the averaged trace. The amplitude was expressed as a percentage of the maximum response as determined by supramaximal electrical stimulation at Erb's point or to the muscle's motor nerve. This is referred to as the 'relative amplitude' (\% max M).

Upon completion of the mapping, sites from which responses (in any of the target muscles) were evoked were restimulated at progressively lower stimulus intensities in order to determine threshold. Threshold was defined as the minimum stimulus intensity required to elicit a response greater than $50 \mu \mathrm{V}$ two out of three times in the relaxed muscle. Similarly, threshold stimulus intensities were determined while the target muscle was contracted.

\section{Data analysis}

An analysis of variance (ANOVA) was used to compare mapping characteristics across muscles and groups. For all tests a probability level of $P<0.05$ was adopted.

\section{Results}

Subjects

Ten individuals with cervical cord lesions (mean age $\pm 1 \mathrm{SD}=35.3 \pm 5.4$ years) and 12 healthy, similarly aged controls (mean age $\pm 1 \mathrm{SD}=31.9 \pm 6.2$ years) participated in the study. All of the subjects with quadriplegia had sustained traumatic injuries and were healthy prior to injury. Five subjects with SCI had complete lesions and the others had partial preservation of sensory or motor function below the lesion level including the lowest sacral segment $(\mathrm{S} 4-5)$. None of the subjects with quadriplegia had normal functional use of their digits, hands, or wrist flexors. The demographic data and clinical findings are summarized in Table 1.

The mapping study was completed for all subjects despite the lengthy recording sessions which approximated 4-6 h. Short latency MEPs following focal transcranial magnetic stimulation applied over the scalp were observed in the relaxed contralateral muscles of $5 / 12$ control subjects $(11 / 36$ muscles tested) and only 2/10 subjects with SCI (3/30 muscles tested), both of whom had complete lesions. The number of scalp sites from which MEPs could be evoked was in all cases less in the SCI subjects $(2-3$ sites) than controls ( $3-6$ sites), and responses were of smaller relative amplitudes $(\% \max \mathrm{M})$. The paucity of motor responses is a significant finding in terms of previous reports although it precluded more detailed analysis of these data.

In contrast, when subjects maintained low level isometric contractions in the target muscles MEPs were readily elicited (control: 32/36 muscles tested; SCI: $24 / 30$ muscles tested). The onset latencies of the MEPs were consistent amongst subjects in the control group and those in the complete and incomplete SCI groups $(\mathrm{F}=1.21, \quad P>0.30)$. The mean response latencies ranged from $11.3 \mathrm{~ms}$ to $15.3 \mathrm{~ms}$.

It should be noted that there was no instance when a MEP was recorded ipsilateral to the side of stimulation. Furthermore, amongst the subjects with SCI there were no visibly detectable muscle contrac-
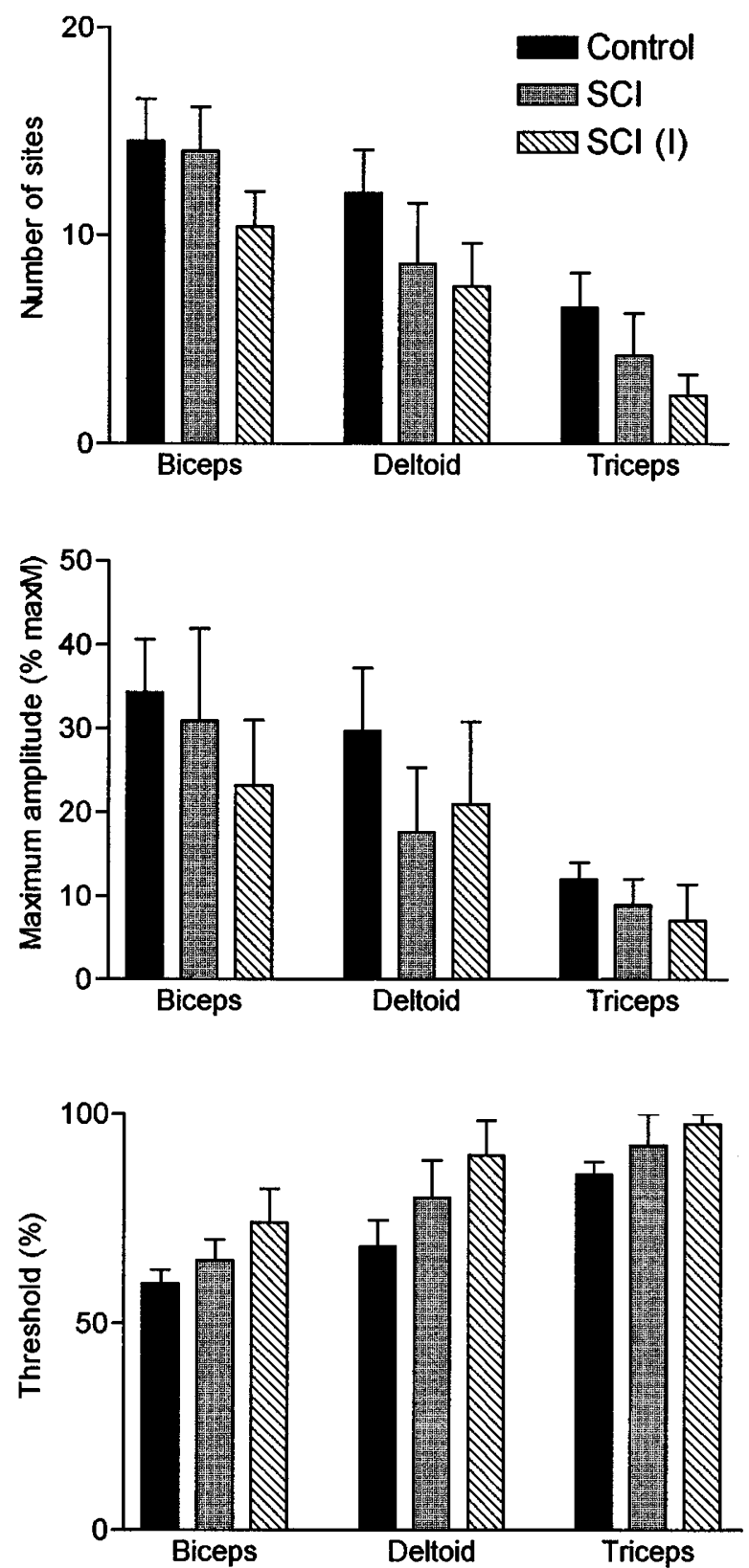

Figure 1 Mean map size (a), maximum MEP amplitudes (b) and threshold stimulus intensities (c) plus 1 SEM as a function of muscle and group. I = incomplete 
tions in the wrist flexors or extensors even though the extensors had some degree of residual function or normal function (see Table 1). In control subjects muscles associated with wrist and finger movements often visibly twitched in response to maximal stimulation.

The number of scalp sites from which MEPs could be elicited (with background contraction) varied depending on the muscle in question $(\mathrm{F}=11.41$,
$P<0.001)$. The largest cortical representations were associated with biceps, then deltoid, with much smaller cortical areas projecting to triceps. The mean size of the cortical representations (expressed as the number of sites) appeared to be smaller amongst patients with SCI and incomplete lesions in particular, although this was not borne out statistically $(\mathrm{F}=2.53, P>0.09)$ (Figure 1a). The clusters of grid points from which responses were evoked in a given muscle were always
Control
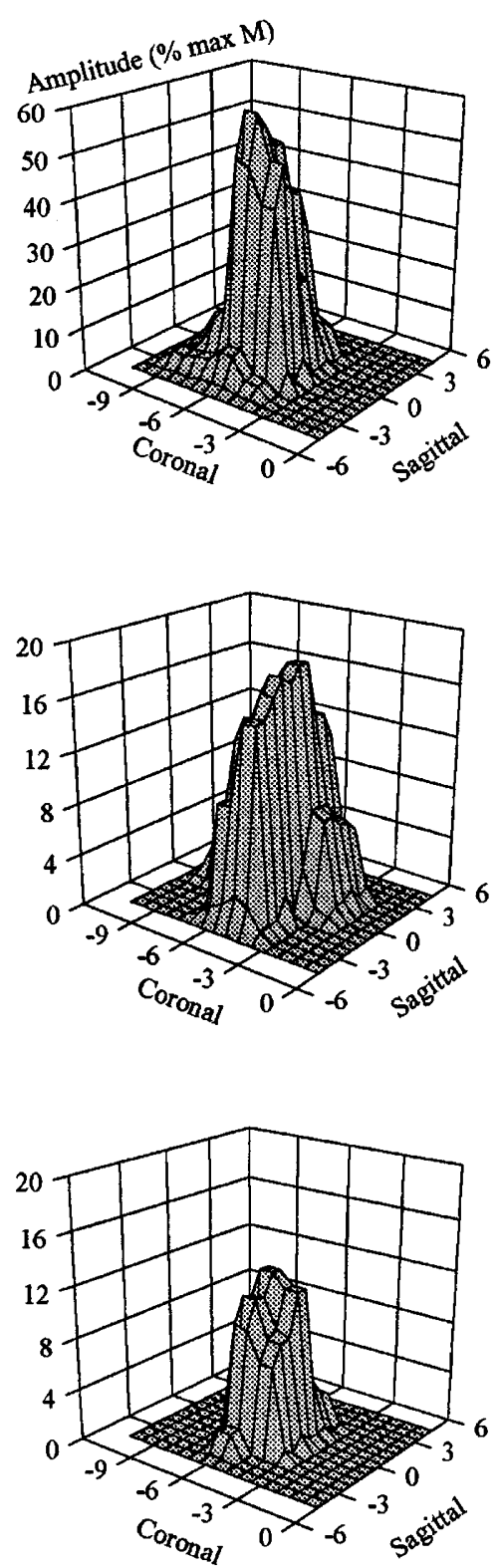

$\mathrm{SCl}$
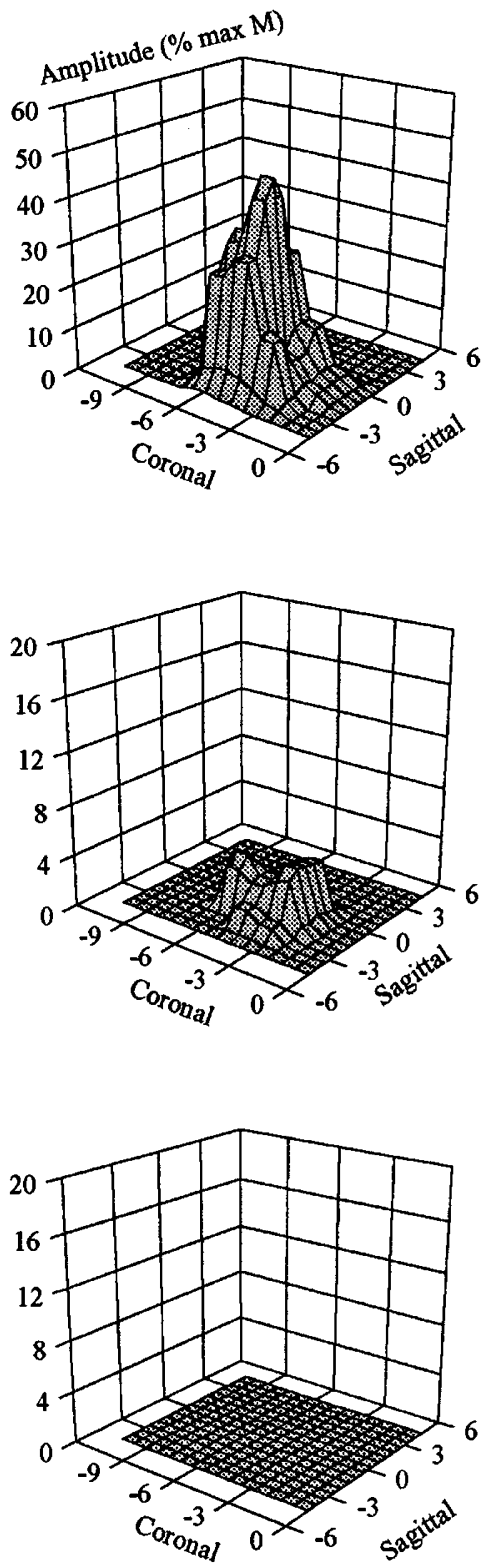

$\mathrm{SCl}(\mathbf{l})$
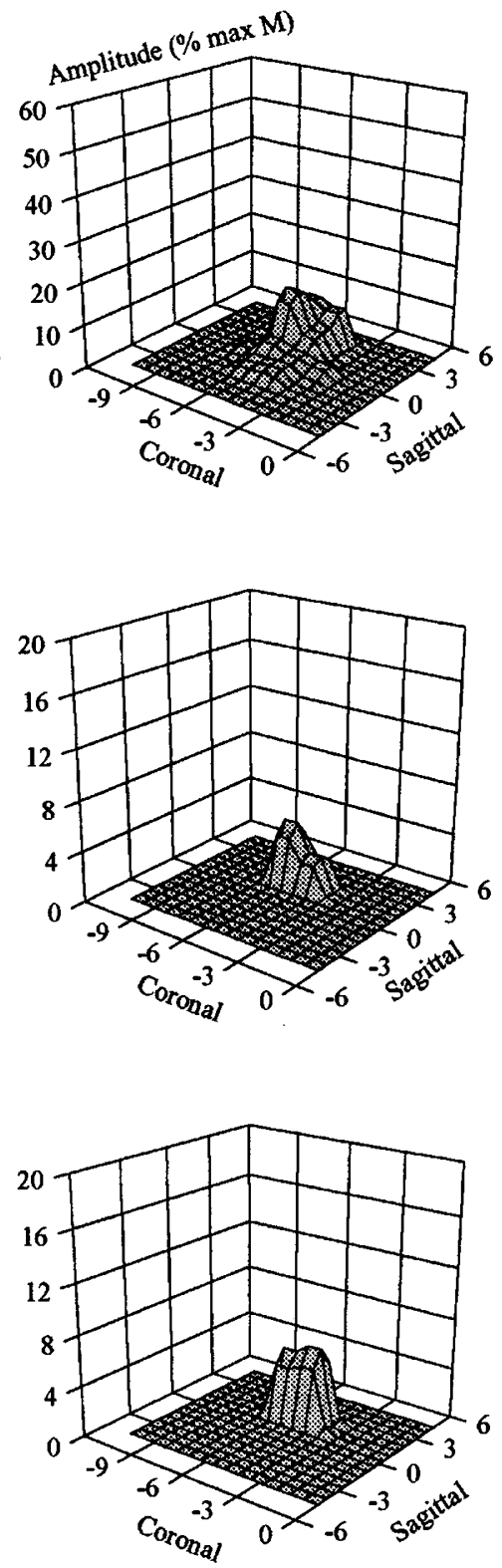

Figure 2 The motor cortical representations of the biceps (top row), deltoid (middle row) and triceps (bottom row) muscles for a single subject from each group in response to maximal stimulation $(100 \%)$. The lesion level for both patients was at C6 which explains the absence of triceps responses when the lesion was complete (centre column). Sagittal and coronal coordinates are in $\mathrm{cm}$ with the vertex, $\mathrm{Cz}$, at 0.0 . Note the different scaling along the axis representing the MEP amplitudes 


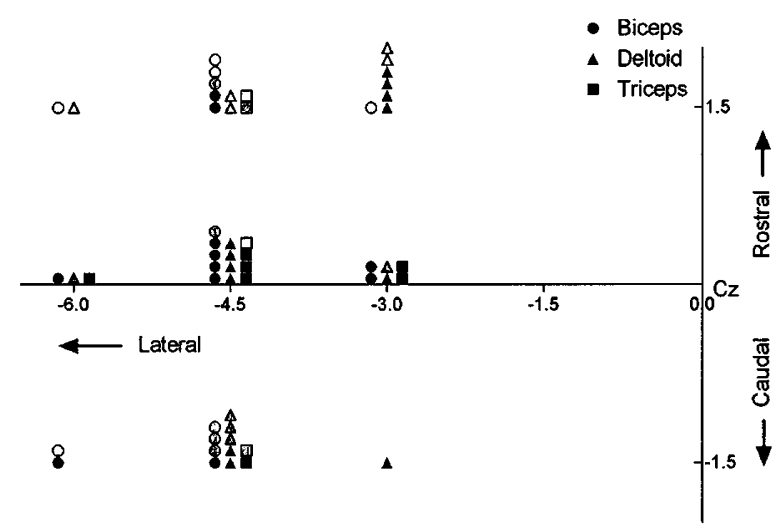

Figure 3 A summary of the optimal sites of stimulation for individual subjects in each group (filled symbols=control subjects; open symbols = SCI subjects; shaded symbols $=$ incomplete SCI subjects. Muscles are represented by the shape of the symbol as illustrated in the legend. Coordinate measures are in $\mathrm{cm}$

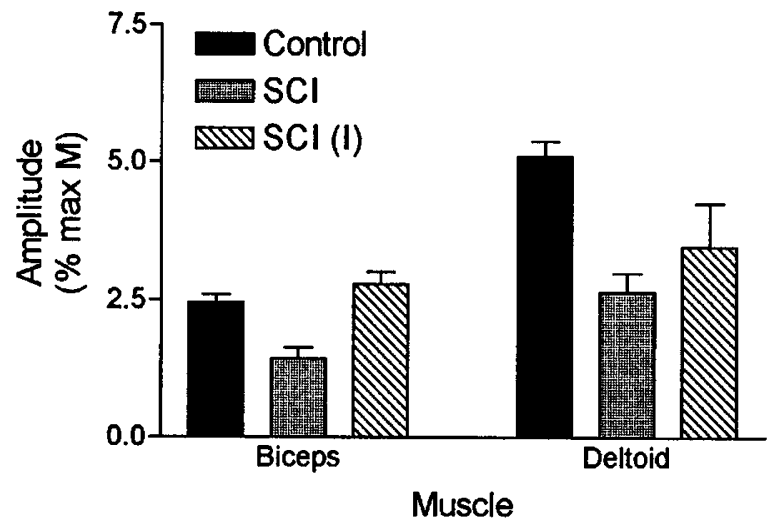

Figure 4 The mean MEP amplitudes (+1 SEM) evoked from the optimal stimulus site at threshold intensities of magnetic stimulation

contiguous and any given subject had only one site, termed the optimal site, from which the largest MEP could be evoked (see Figure 2). Surrounding this site were points from which smaller MEPs were elicited. The location of the optimal site varied between subjects and, from observation, appeared to vary between groups but less so between muscles (Figures 2 and 3). Figure 2 shows extensive overlap between cortical representations of the three muscles examined.

The mean maximal MEP amplitude (normalized to max M) elicited following maximal stimulation applied to the optimal scalp site were largest for the biceps muscle and smallest for triceps $(\mathrm{F}=4.34, P<0.02)$. There were no group differences $(\mathrm{F}=0.70, P>0.50)$ although the MEPs obtained from the SCI groups tended to be smaller in amplitude (Figures $1 \mathrm{~b}$ and 2). Of note is that there were no differences in the magnitude of the maximal $\mathrm{M}$ waves obtained across the three groups $(\mathrm{F}=1.17, P>0.31)$ although they were largest for the biceps muscle, followed by deltoid then triceps $(\mathrm{F}=10.05, P<0.003)$.

Main effects of group $(\mathrm{F}=3.44, \quad P<0.05)$ and muscle $(\mathrm{F}=8.84, \quad P<0.001)$ were observed with respect to the stimulus threshold required to elicit a motor response. Within a projection area, the distribution of the threshold values generally revealed a 'best point' from which a MEP was evoked at comparatively low intensity. This point was the same as or immediately adjacent to the optimal site defined above. Thresholds for activating the biceps muscle were lower than the intensities required to evoke motor responses in the other muscles (Figure 1c). In terms of group, subjects with incomplete SCI required higher stimulus levels to activate proximal upper extremity muscles than control subjects, but not subjects with complete lesions.

The higher activation thresholds in the incomplete SCI group confounds the interpretation of the amplitude data due to the known positive relationship between MEP amplitude and stimulus intensity (see Rossini ${ }^{16}$ ). In control subjects, maximal stimulator output was 'relatively' higher than in subjects with incomplete SCI owing to the larger differential between threshold and $100 \%$ stimulus intensities; therefore, MEP amplitudes would be expected to be larger in control subjects under conditions of maximal stimulation. Analysing the MEP amplitudes elicited in response to threshold stimulation reveals both a similarity between groups $(\mathrm{F}=1.618, P>0.21)$ and between the biceps and deltoid muscles $(\mathrm{F}=3.57$, $P>0.06)$ although the pattern was somewhat altered from that observed when stimulating at $100 \%$ intensity (contrast Figure 4 and Figure 1b). The MEPs from triceps were not reanalysed in this way since the thresholds closely approximated the maximal stimulator output.

\section{Discussion}

Focal transcranial magnetic stimulation readily evoked short latency contralateral MEPs in the gently contracted proximal upper extremity muscles in all subjects tested but rarely in relaxed muscles. In control and both SCI groups, biceps and deltoid motoneuron pools were more easily and extensively recruited than triceps motoneurons under active conditions. This pattern of activity (ie biceps $>$ deltoid $>$ triceps) has been linked to the relative 'interest' of the motor cortex in these motoneuron pools ${ }^{17,18}$ and is consistent with the known pattern of projections of the rapidly conducting primate corticospinal tract. ${ }^{19}$

Corticospinal reorganization in humans has been inferred on the basis of abnormally elevated corticospinal excitability and enlarged cortical areas projecting to specific motoneuron pools in subjects at rest with chronic central ${ }^{10,11}$ or peripheral lesions. ${ }^{7-9}$ Topka et $a l^{11}$ reported lower thresholds of corticospinal neurons projecting to relaxed muscles whose 
innervation level was immediately rostral to the thoracic spinal cord lesion. They and others ${ }^{10}$ have illustrated dramatically enlarged cortical representations of spared muscles as compared to homologous muscles in control subjects; likely reflecting a take over of cortical areas associated with muscles innervated by roots caudal to the lesion. In the present study MEPs were observed in only two subjects with SCI (three muscles) and five control subjects (11 muscles) in the relaxed contralateral muscles. Furthermore there was no instance when the cortical representation area was larger in a subject with a SCI than a control subject suggesting that detectable reorganizational changes are not necessarily a sequela of chronic SCI.

Previous studies involving SCI patients (using the same stimulator and coil) have recorded large amplitude MEPs following stimulation of numerous scalp sites while subjects were at rest. Levy et al ${ }^{10}$ revealed expanded motor representations of the relaxed biceps and deltoid muscles in two patients with quadriplegia in response to stimulation at intensities comparable to that required to elicit responses in the muscles of the hand in control subjects. This raises the possibility that corticospinal excitability was low in our subjects. Activation thresholds are known to be higher in proximal than distal muscles..$^{20,21}$ and in the case of control subjects, visible contractions in relaxed forearm and hand muscles were frequently observed at intensities lower than that required to record a response from a more proximal muscle. In SCI this did not occur and threshold stimulation was always $100 \%$ of the stimulator output. It is possible that in SCI the likelihood of observing a MEP may be diminished due to temporal dispersion associated with nonuniform central conduction through dysmyelinated axons. In human SCI, macroscopic cord lesions can extend $1-5 \mathrm{~cm}$ longitudinally ${ }^{22}$ and microscopic lesions including myelin sheath disruption can occur throughout $4-5$ segments adjacent to the lesion level. ${ }^{23}$ The extent of the pathology in our subjects was not known.

Cohen et $a l^{24}$ were also unable to demonstrate cortical reorganization in one subject with a longstanding SCI which they associated with a lack of participation in extensive therapy. The patients in Levy et $a l^{10}$ study were documented to have had extensive therapy including biofeedback training. Although the rehabilitation history of the patients in the present study was not known they did present with varied functional levels although this did not seem to bear on the findings. Perhaps specific intervention strategies may be required to produce detectable cortical reorganization. In animals, additional sensory stimulation has been associated with the enlargement of cortical receptive fields, ${ }^{25,26}$ muscle activation has been shown to expand the size of the motor representation, ${ }^{27}$ and shifts in motor cortical topography following induced CNS lesions of about $670 \mu \mathrm{M}$ are considered substantial. ${ }^{28}$ In humans, changes of corresponding magnitudes would be extremely difficult to detect (if at all possible) with the current noninvasive techniques ${ }^{29}$ and as such they are limited to uncovering more robust reorganization.

Isometric voluntary contraction has a facilitatory effect on responses to motor cortical stimulation ${ }^{30}$ and as such can be used to enhance low amplitude MEPs or reveal otherwise undetectable MEPs. With contraction, motor responses were elicited from all subjects with SCI as well as controls following stimulation of several scalp sites. Motor maps defined at rest or during contraction differ in terms of the location of the optimal site of stimulation and maps increase their size under contracted conditions. ${ }^{31}$ Such changes have been attributed to the effects of muscle afferent activity on corticomotor output as well as changes in cortical excitability. ${ }^{31,32}$ Following complete and incomplete SCI optimal sites of stimulation tend to be rostral and caudal respectively to the optimal site of control subjects located lateral to the vertex. This finding suggests differences in the spatial properties or pattern of cortical excitability which may result from altered afferent input or variations in contraction strategy (cf Wilson et $a l^{31}$ ). In all cases though there is overlap between the cortical areas projecting to the proximal upper extremity muscles.

The size of the motor cortical representations and the maximum MEP amplitudes were smaller (though not statistically different) amongst subjects with SCI and those with incomplete lesions in particular. The size of the cortical map is necessarily related to corticospinal excitability in response to a given stimulus. Since the activation thresholds tended to be higher in subjects with SCI and significantly so in those with incomplete lesions, the relative map sizes may have been underestimated in the patient group. It is well known that with higher intensity stimulation progressively larger MEPs are produced ${ }^{16,21}$ as a greater proportion of the corticomotoneuronal pool is activated and a greater number of descending volleys or I-waves ${ }^{27}$ spatiotemporally summate at the level of the alphamotoneurons. When activation thresholds are significantly higher (lower) for a given subject group, then it follows that the map sizes and MEP amplitudes would consequently be smaller (larger). By extension, differences may be less apparent if the intensity of stimulation were relative to threshold rather than an absolute maximum (ie $100 \%$ ). The increased similarity of MEP amplitudes across groups at threshold stimulation supports this argument (see Figures 1 and 3).

Although it may not be appropriate to contrast motor maps generated at rest with those produced during contraction ${ }^{33}$ it is however notable that there was no evidence of motor area enlargement among the subjects with SCI during tonic contraction as might have been predicted on the basis of previous studies. Ridding and Rothwell ${ }^{33}$ have shown in two subjects with unilateral amputations that evidence of larger motor representations to muscles proximal to the stump compared to their homologous counterparts 
disappeared when the maps were constructed during contraction. The explanation for the disappearance is unclear although it suggests than an active state may mask the presence of reorganization during rest. Weiller et $a l^{12}$ used positron emission tomography to reveal increased areas of cortical activation in patients recovering from acute or chronic motor stroke relative to control subjects. They reported greater activation of structures belonging to motor and sensorimotor pathways which they presented as evidence of cortical reorganization following stroke. The patterns these authors observed were present at rest as well as during activation. In combination these findings suggest that the utility of examining cortical reorganization in the presence of facilitation may vary depending on whether the damage is peripheral or central.

The findings of this study fail to support previous reports ${ }^{10,11}$ of expanded motor cortical representations to relaxed muscles innervated by roots rostral to the lesion in SCI patients. During tonic low level contraction the activation thresholds are highest for subjects with incomplete SCI and lowest among control subjects. This was accompanied by smaller motor cortical representations in SCI. While it is possible that the variable degrees of sensorimotor sparing below the designated lesion level may impact on the excitability of corticomotoneurons or their afferents, careful examination of the data did not reveal any apparent associations between an outcome measure and functional level. For example, MEP amplitudes and map sizes for biceps and deltoid muscles were comparable for an individual with a complete C6 lesion and a subject with an incomplete C7 SCI and a ' $C$ ' classification on the ASIA impairment scale. ${ }^{14}$ In view of the previous literature relating to cortical reorganization following SCI and the findings of the present study there is a need for further investigation to determine the association of pathology and specific rehabilitation intervention strategies with the occurrence or extent of motor cortical expansion.

\section{Acknowledgements}

This research was supported by the Rick Hansen Man in Motion Legacy Fund (Grants 91-12 and 92-06), the Easter Seal Research Institute, and the Botterell Foundation (Queen's University).

\section{References}

1 Mortifee P, Stewart H, Schulzer M, Eisen A. Reliability of transcranial magnetic stimulation for mapping the human motor cortex. Electroencephalography and Clinical Neurophysiology 1994; 93: $131-137$.

2 Cohen LG, Hallett M. Noninvasive mapping of human motor cortex. Neurology 1988; 38: $904-909$.

3 Wassermann EM, McShane LM, Hallett M, Cohen LG. Noninvasive mapping of muscle representations in human motor cortex. Electroencephalography and Clinical Neurophysiology 1992; 85: $1-8$.
4 Penfield W, Boldrey E. Somatic motor and sensory representation in the cerebral cortex of man as studied by electrical field stimulation. Brain 1937; 60: $389-443$.

5 Woolsey CN, Eriksen TC, Gilson WE. Localization in somatic sensory and motor areas of human cerebral cortex as determined by direct recording of evoked potentials and electrical stimulation. Journal of Neurosurgery 1979; 51: 476-506.

6 Pascual-Leone A et al. Modulation of motor cortical outputs to the reading hand of Braille readers. Annals of Neurology 1993; 34: $33-37$.

7 Cohen LG, Bandinelli S, Findley TW, Hallett M. Motor reorganization after upper limb amputation in man. A study with focal magnetic stimulation. Brain 1991; 114: 615-627.

8 Cohen LG et al. Magnetic stimulation of the human cerebral cortex, an indicator of reorganization in motor pathways in certain pathological conditions. Journal of Clinical Neurophysiology 1991; 8: $56-65$.

9 Fuhr $\mathrm{P}$ et al. Physiological analysis of motor reorganization following lower limb amputation. Electroencephalography and Clinical Neurophysiology 1992; 85: 53-60.

10 Levy WJ, Amassian VE, Traad M, Cadwell J. Focal magnetic coil stimulation reveals motor cortical system reorganized after traumatic quadriplegia. Brain Research 1990; 510: 130 134

11 Topka H, Cohen LG, Cole RA, Hallett M. Reorganization of corticospinal pathways following spinal cord injury. Neurology 1991; 41: $1276-1283$.

12 Weiller C et al. Individual pattern of functional reorganization in human cerebral cortex after capsular infarction. Annals of Neurology 1993; 33: 181-189.

13 Weiller C et al. Function reorganization of the brain in recovery from striatocapsular infarction in man. Annals of Neurology 1992; 31: $463-472$.

14 American Spinal Injury Association (ASIA) Standards for Neurological and Functional Classification of Spinal Cord Injury. Rev. 1992.

15 Jasper HH. Report of the committee on methods of clinical examination in electroencephalography. APPENDIX: The ten twenty electrode system of the international federation. Electroencephalography and Clinical Neurophysiology 1957; 10: $370-375$.

16 Rossini PM. Methodological and physiological aspects of motor evoked potentials. In: Rossini PM, Maugiere F (eds.) New Trends and Advanced Techniques in Clinical Neurophysiology. Electroencephalography Supplement. Elsevier Science Publishers, B.V. 1990, pp 124-133.

17 Lemon RN, Mantel GWH, Muir RB. Corticospinal facilitation of hand muscles during voluntary movement in the conscious monkey. Journal of Physiology 1986; 381: $497-527$.

18 Palmer E, Ashby P. Corticospinal Projections to Upper Limb Motoneurones in Humans. Journal of Physiology 1992; 448: $397-412$.

19 Phillips CG, Porter R. The pyramidal projection to motoneurones of some muscle groups of the baboon's forelimb. Progress in Brain Research 1964; 12: 222 - 245.

20 Rothwell JC et al. Motor cortex stimulation in intact man. 1 General characteristics of EMG responses in different muscles. Brain 1987; 110: $1173-1190$.

21 Brouwer B, Ashby P. Corticospinal projections to upper and lower limb spinal motoneurons in man. Electroencephalography and Clinical Neurophysiology 1990; 76: 509-519.

22 Bedbrook GM. Some pertinent observations on the pathology of traumatic spinal paralysis. Paraplegia 1963; 1: 215-217.

23 Holmes G. Spinal injuries of warfare. British Medical Journal 1915; ii: $769-774$.

24 Cohen LG et al. Topographic maps of human motor cortex in normal and pathological conditions: mirror movements, amputations, and spinal cord injuries. Electroencephalography and Clinical Neurophysiology 1991; 43 (Suppl): 36-50.

25 Jenkins WM et al. Functional Reorganization of Primary Somatosensory Cortex in Adult Owl Monkeys after Behaviorally Controlled Tactile Stimulation. Journal of Neurophysiology 1990; 63: $82-104$. 
26 Recanzone GH, Allard TT, Jenkins WM, Merzenich MM Receptive field changes induced by peripheral nerve stimulation in SI of adult cats. Journal of Neurophysiology 1990; 63: 1213 1225 .

27 Humphrey DR, Qui XQ, Clavel P, O'Donoghue DL. Changes in forelimb motor representation in rodent cortex induced by passive movements. Society for Neuroscience Abstracts 1990; 16: 422 .

28 Asanuma C. Mapping movements within a moving motor map. Trends in Neuroscience 1991; 14: $217-218$.

29 Brasil-Neto JP et al. Topographic mapping of the human motor cortex with magnetic stimulation: factors affecting accuracy and reproducibility. Electroencephalography and Clinical Neurophysiology 1992; 85: $9-16$.
30 Maertens de Noordhout A, Pepin JL, Gerard P, Delwaide PJ. Facilitation of responses to motor cortex stimulation: effects of isometric voluntary contraction. Annals of Neurology 1992; 32: $365-370$.

31 Wilson SA, Thickbroom GW, Mastaglia FL. Comparison of the magnetically mapped corticomotor representation of a muscle at rest and during low-level voluntary contraction. Electroencephalography and Clinical Neurophysiology 1995; 97: 246-250.

32 Berardelli A et al. The site of facilitation of the response to cortical stimulation during voluntary contraction in man. Journal of Physiology 1984; 360: 52P.

33 Ridding MC, Rothwell JC. Reorganisation in human motor cortex. Canadian Journal of Physiology and Pharmacology 1995; 73: $218-222$. 